\title{
ABSORPTION OF 19.7-MC/S RADIATION IN HII REGIONS
}

\author{
C. A. SHAIN \\ Radiophysics Laboratory, Commonwealth Scientific and Industrial \\ Research Organization, Sydney, Australia
}

1. INTRODUCTION

The main program of the Sydney $19.7-\mathrm{Mc} / \mathrm{s}$ cross is devoted to the observation of the brightness distribution in a strip, extending some 10 degrees on either side of the galactic equator, the particular interest being the detection of absorption in $\mathrm{H}$ II regions. This program is well advanced, but since observing time is limited by ionospheric absorption and interference, records are not yet available for a number of galactic crossings. The detailed analysis of the observations has just begun, and will take some time. In the meantime a rough analysis of the data has been made in order to get a general picture of what might be expected, and this paper gives an outline of the results. The application of various instrumental corrections and the detailed analysis of ionospheric effects may subsequently necessitate some changes in the numbers involved in the calculations, but it is expected that the general conclusions will still hold.

As described in an earlier paper [1], the observations show a trough around a large part of the galactic equator, due to the large-scale distribution of ionized hydrogen in the Galaxy. At many points near the galactic plane, and also in some higher latitude directions, there is additional absorption due to comparatively nearby (2 kiloparsecs or less) $\mathrm{H}$ il regions-many of them well known from optical observations. Fig. 1 shows crossings of the galactic plane at two longitudes near known $\mathrm{H}$ il regions (the positions of those observed by Gum [2] are shown by crosses). The aerial has a beamwidth of about 1.4 and the usual operation is to switch, at 12-second intervals, to five separate declinations in turn, so that each record, as in Fig. 1, gives a scan over a range of rather more than 2 degrees in declination. In the upper record in Fig. 1 we see the strong absorption around $M 8$ and $M 20$, and in the lower record the absorption near Stromlo 66 and 67.

The temperatures observed in these directions are made up of several components: thermal emission from the $\mathrm{H}$ II region itself; nonthermal background radiation transmitted (with attenuation) through the $\mathrm{H}$ il region or entering the aerial beam around the sides; and foreground nonthermal radiation, emitted between the $\mathrm{H}$ il region and the sun. We shall first consider the observations of some of the "nearby" $H$ II regions, with a view to estimating the foreground emission in several directions near the sun, and then see how the general distribution of absorption fits in with the current ideas on galactic structure. Finally, we note that it is possible to fix the distances of some nonthermal sources with respect to absorbing $\mathrm{H}$ II regions. 

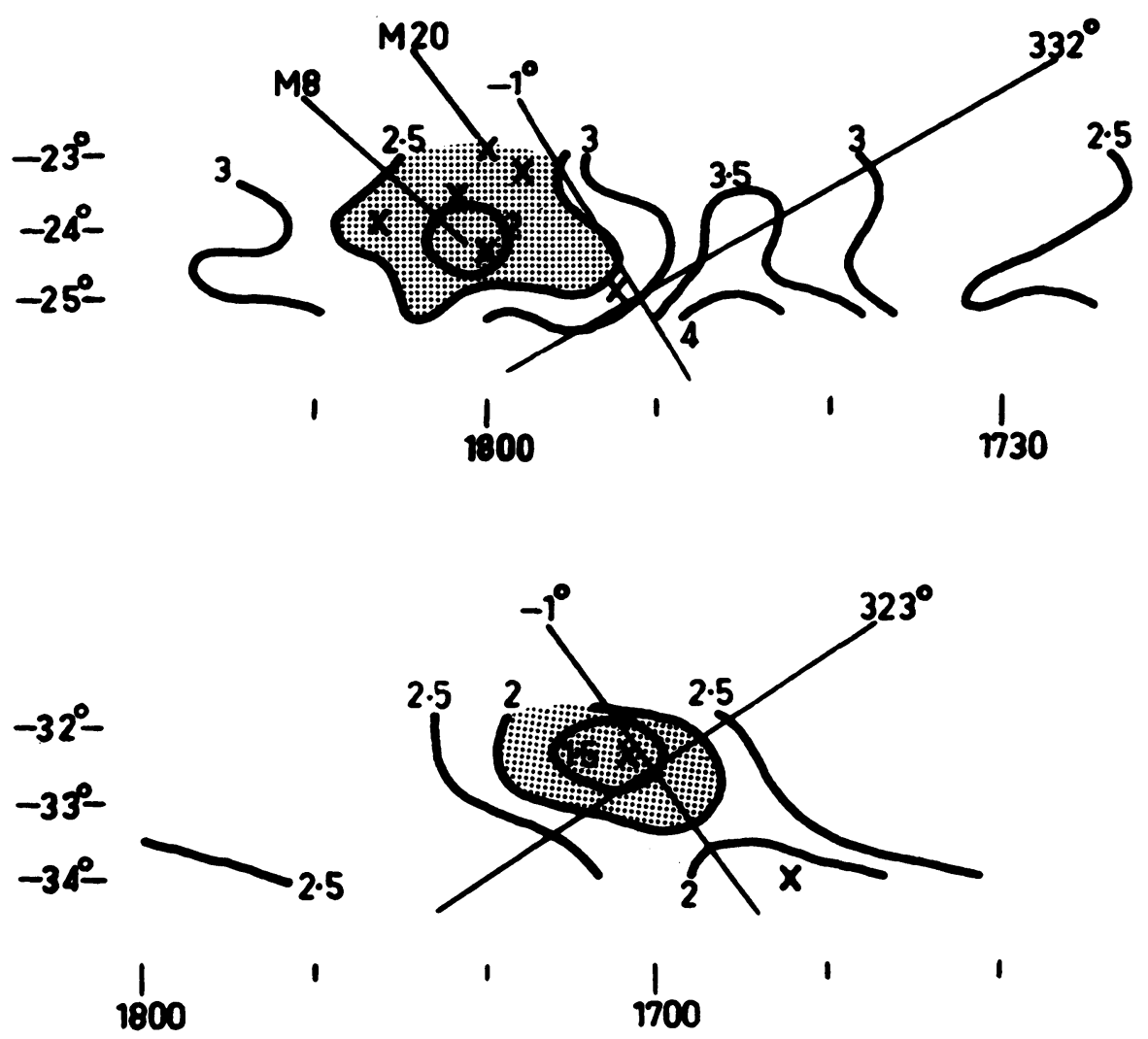

FIg. 1. Two records showing the observation of $\mathrm{H}$ II regions in absorption. The crosses mark the positions of $\mathrm{H}$ iI regions observed optically.

\section{OBSERVATIONS OF NEARBY H II REGIONS}

Let $T_{e}$ be the electron temperature of the $\mathrm{H}$ In region, $\alpha$ the fraction of background radiation incident on the $\mathrm{H}$ II region that is transmitted through it, and $\beta$ the fraction that enters the aerial beam around the sides. $T_{1}$ is the temperature observed in a neighboring "clear" area of the sky, $T_{2}$ the temperature observed in the direction of the $\mathrm{H}$ II region, and $T_{3}$ the foreground component of $T_{2}$. Then

$$
T_{2}=(1-\alpha)(1-\beta) T_{e}+[\beta+\alpha(1-\beta)]\left(T_{1}-T_{3}\right)+T_{3},
$$

from which we can estimate the foreground emission

$$
T_{3}=\left[T_{2}-(1-\alpha)(1-\beta) T_{e}-(\beta+\alpha-\alpha \beta) T_{1}\right] /(1-\beta-\alpha+\alpha \beta) .
$$

As was shown in [1], an emission measure (EM) of 800 gives an optical depth of unity for $19.7 \mathrm{Mc} / \mathrm{s}$ radiation so that for any but the optically faintest $\mathrm{H}$ II regions $\alpha$ will be quite small. On the other hand, optical $\mathrm{H}$ il regions range in size from a few minutes of arc to several degrees; unless the $\mathrm{H}$ il region fills an appreciable fraction of the aerial beam, $\beta$ is near 1. 
Table I presents the results for several $\mathrm{H}$ II regions. The identification numbers and distances (unless stated below) are taken from Gum [2].

\section{TABLE I}

\begin{tabular}{|c|c|c|c|c|c|c|}
\hline NONTHERMAL & EMISSION & BETWEEN & THE SUN & AND “ NE & $\mathrm{BBY}$ " $\mathrm{H} \mathrm{II}$ & REgIONS \\
\hline H II Region & $\begin{array}{l}\text { Distance } \\
\text { (pc) }\end{array}$ & $\alpha$ & $\beta$ & $\begin{array}{c}T_{1} \\
\left({ }^{\circ} \mathrm{K}\right)\end{array}$ & $\begin{array}{c}T_{2} \\
\left({ }^{\circ} \mathrm{K}\right)\end{array}$ & $\begin{array}{r}T_{3} \\
\left({ }^{\circ} \mathrm{K}\right)\end{array}$ \\
\hline Stromlo 67 & 600 & 0.1 & 0.25 & 275,000 & 130,000 & 50,000 \\
\hline Stromlo 55 & 1250 & 0 & 0.4 & 200,000 & 120,000 & 55,000 \\
\hline $\begin{array}{l}\text { Stromlo } 73 \\
\text { ( }(\mathrm{Oph})\end{array}$ & 150 & $<0.35$ & 0 & 200,000 & 130,000 & $>86,000$ \\
\hline$\underset{\text { (part) }}{\text { Stromlo }} 12$ & 200 & 0.02 & 0.25 & 80,000 & 40,000 & 15,000 \\
\hline$\tau$ Scorpii & 200 & 0.6 & 0 & 200,000 & 150,000 & 26,000 \\
\hline $\begin{array}{l}\text { Stromlo } 72 \\
(\text { M 8) }\end{array}$ & 1300 & 0 & 0.5 & 300,000 & 200,000 & 80,000 \\
\hline Stromlo 83 & 2300 & 0 & $>0.5$ & 160,000 & 100,000 & $<30,000$ \\
\hline
\end{tabular}

(M 16)

Stromlo 84

Stromlo $67\left(323^{\circ} 4,-1.3\right)$ : Listed as faint in the catalog, although Gum (unpublished data) has estimated the emission measure as several thousand. Westerhout [3] deduces an EM of 2000 so that $\alpha$ is about 0.1 . All observations agree that the shape is regular and the diameter is 2 degrees. Estimates of the distance vary; the value is Johnson's [4].

Stromlo 55 (part) $\left(309^{\circ},-1^{\circ}\right)$ : Part of the large loop, equivalent to a strip about 3 degrees long by 1 degree wide, giving $\beta=0.4$.

Stromlo $73\left(\zeta\right.$ Ophiuchi nebula) $\left(334^{\circ},+23^{\circ}\right)$ : This nebula covers an area about 10 degrees in diameter. It appears patchy, which is probably caused by foreground obscuration. The $19.7-\mathrm{Mc} / \mathrm{s}$ records suggest that the absorption is practically uniform over a large area; we can therefore put $\beta=0$. In estimating $\alpha$, we note that Sharpless and Osterbrock [5] estimated the EM as about 500, but other radio observations suggest that the EM is somewhat higher than this; Blythe [6] from his 38-Mc/s observations estimates the EM as 1800 . As a conservative estimate we say that the EM is greater than 800 , so that $\alpha=0.35$.

Stromlo 12 (part) $\left(230^{\circ},-7^{\circ}\right)$ : A detailed study of the Vela-Puppis region has been made by Rishbeth [7], who notes a well-marked absorption feature at this position. The part of the nebula concerned is quite bright, with an effective diameter of 2 degrees, and $\alpha$ is estimated to be not greater than 0.02 . This is probably one of the nearer parts of the nebula, so that we have taken the distance as 50 parsecs less than that of $\gamma$ Velorum.

Nebula surrounding i Scorpii $\left(328^{\circ},+10^{\circ}\right)$ : The diameter is about 5 degrees [1, 8]. We have taken the EM as 400 , which again is probably rather lower than the true value.

Stromlo 72 (M 8) (333:7, - 2.6): This is very bright. Even including other fainter nebulosity at the same distance, $\beta$ can hardly be less than 0.5 . The distance is that given by Morgan, Whitford, and Code [9].

Stromlo 83 (M 16) and $84\left(345^{\circ},-0.5\right)$ : Both are so bright that $\alpha=0 . \quad \beta$ cannot be less than 0.5. This distance is also given in [9]. 
If the nonthermal emission per unit distance were constant throughout the part of the Galaxy concerned, there should be a linear relation between $T_{3}$ and distance to the respective nebulae. The data from Table I are plotted in Fig. 2, on which is included a line having a slope of $100^{\circ} \mathrm{K}$ per parsec;

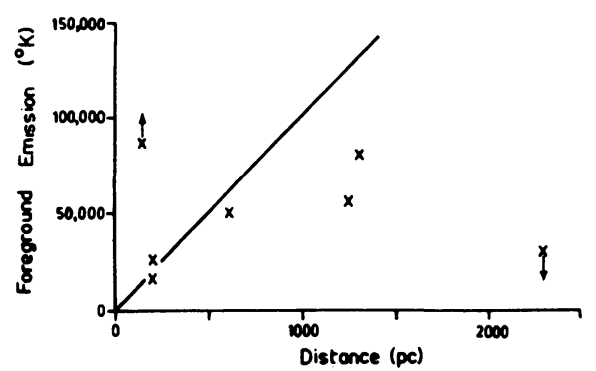

FIG. 2. Foreground temperature versus distance to $\mathrm{H}$ iI regions. this corresponds to the value of about $2{ }^{\circ} \mathrm{K}$ per parsec at $85.5 \mathrm{Mc} / \mathrm{s}$, obtained by Mills [10] for the emission within a spiral arm. The foreground temperatures should rise with increasing distance from the sun until $\mathrm{H}$ II regions that lie outside the local spiral arm are observed. Fig. 2 suggests that a limiting value of about 50,000 ${ }^{\circ} \mathrm{K}$ is reached at a distance less than 600 parsecs. The data are insufficient to determine the real shape of the curve but an initial slope of $100^{\circ} \mathrm{K}$ per parsec would be suitable. It seems therefore that we have an independent line of evidence supporting Mills's deduction that the discoidal component of the galactic radiation is practically confined to the spiral arms.

Some of the scatter of the points in the figure is owing to the uncertainties in the radio and optical data, but there are also, no doubt, real variations in the emission in different directions. Also, it should be noted that any undetected foreground $\mathrm{H}$ II will have the effect of reducing the apparent value of $T_{3}$. This may explain the low point for M 16 compared with the 1300 parsec points, but to explain in this way the whole deficiency of foreground temperatures for the furthermost $\mathrm{H}$ II regions would require foreground $\mathrm{H}$ II with such large EM ( 2000) that it would not have escaped optical detection.

The outstanding point in Fig. 2 is the one for the $\zeta$ Ophiuchi nebula. The foreground temperature here is over five times the expected value for such a short distance. It will be remembered that a conservative EM value was taken for the calculation, so that $T_{3}$ could be even higher; the upper limit is the value of $T_{2}, 130,000^{\circ} \mathrm{K}$. It is therefore suggested that in this direction there is a comparatively strong source of emission quite near the sun. It may be associated with the $\mathrm{H}$ II region itself, or perhaps with the Ophiuchus dark nebula. However, it cannot be too extensive, because the point for $\tau$ Scorpii is not unduly high. The excess radiation amounts to $60,000{ }^{\circ} \mathrm{K}$, and we might guess the linear dimensions to be about 50 parsecs. If placed in the next spiral arm closer to the galactic center it would appear on the $85.5-\mathrm{Mc} / \mathrm{s}$ cross records as a source near the galactic plane with an angular size of just under one degree and producing an excess aerial temperature of about $1000^{\circ} \mathrm{K}$. Many such sources are observed.

\section{LARGE-SCALE ABSORPTION IN THE GALAXY}

To get a general picture of the absorption observed in the Galaxy, before drawing up accurate contours, the temperatures observed when the aerial 
was directed to galactic latitudes +3 and -1 degrees were read off all the available records covering longitudes from about 290 to 10 degrees. The resulting variation of temperature with longitude is shown in Fig. 3. In addition, for the few records available between longitudes 180 and 290 degrees the temperatures were read at latitude 0 degrees; the range of longitudes between 224 and 242 degrees had already been reduced in detail by Rishbeth [7].

The most noticeable feature of the curves, especially for latitude -1 degree, is the great variability of temperature with longitude. Comparison with Gum's map [2] shows that, apart from the minimum at 328 degrees (corresponding to $17 \mathrm{~S} 2 \mathrm{~A}$ ), all the deep minima on the -1 -degree curve and the minimum at 310 degrees on the +3-degree curve coincide with regions of optical $\mathrm{H}$ il emission. Some of these regions have already been considered in the earlier section of the paper. The pronounced dip between 350 and 8 degrees, and the shallower dip between 338 and 350 degrees, are also

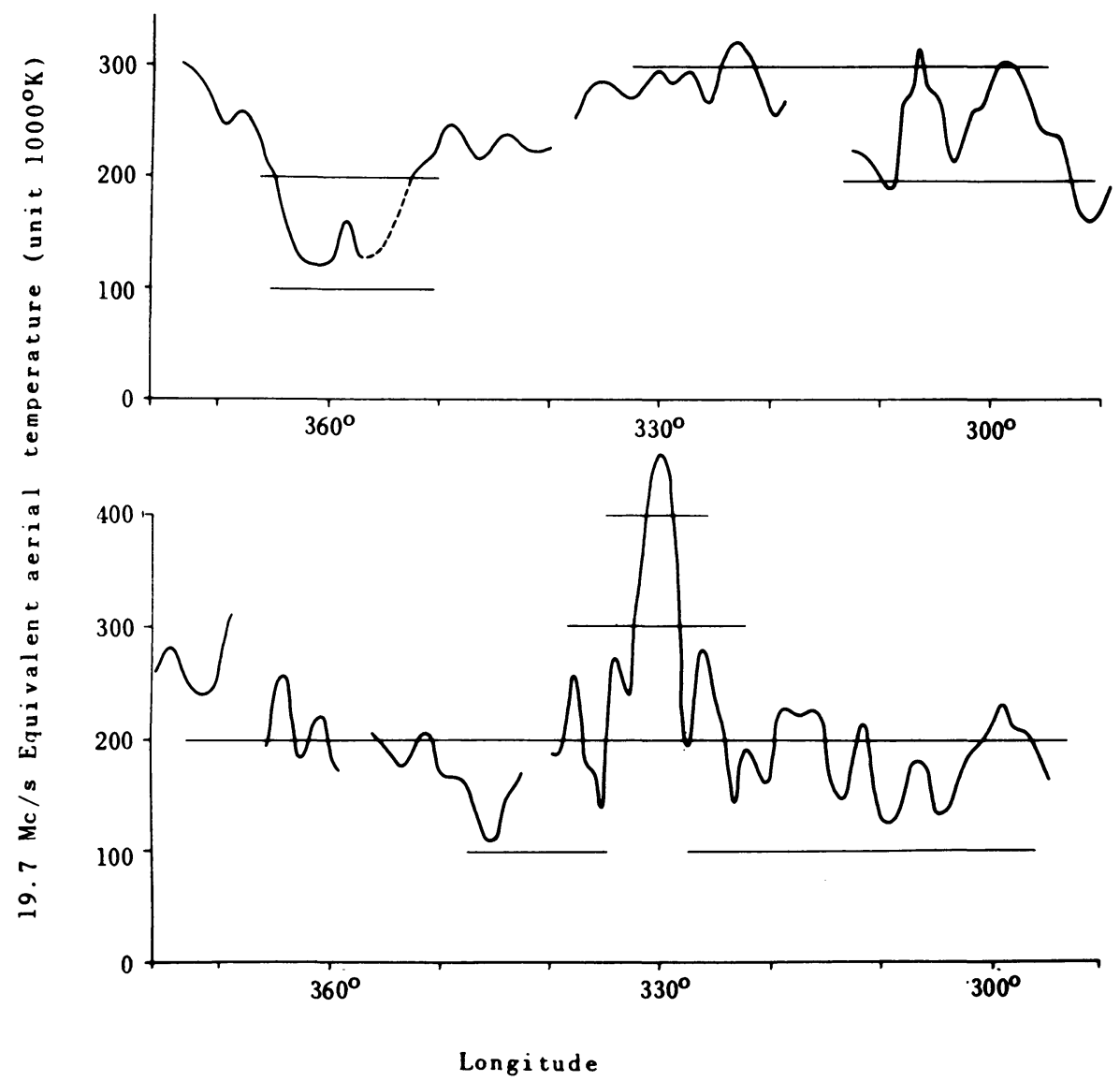

Fig. 3. 19.7-Mc/s equivalent aerial temperature versus longitude for latitudes +3 degrees (top) and -1 degree (bottom). 
due to localized $\mathrm{H}$ II absorption. Westerhout [3] has shown that at these longitudes the thermal component of the temperatures observed at $1390 \mathrm{Mc} / \mathrm{s}$ extends to further than +3 degrees with brightness that would account for the $19.7-\mathrm{Mc} / \mathrm{s}$ absorption if the $\mathrm{H}$ il were near the sun.

All these localized absorbing regions are within about 2 kiloparsecs of the sun; forming part of what Gum has termed an "inter-arm link"; the second point of interest about the -1-degree curve of Fig. 3 is that between these $\mathrm{H}$ II regions the temperatures are still generally less than those observed at +3 degrees. At higher frequencies the reverse is the case, and it is interesting to compare the $19.7-\mathrm{Mc} / \mathrm{s}$ temperatures with those observed by Mills and his collaborators [11] at $85.5 \mathrm{Mc} / \mathrm{s}$.

Fig. 4 shows the variation with longitude of the ratio $T_{19.7} / T_{85.5 .}$ In constructing this figure, the ratio was computed only for those longitudes where there was no deep $19.7-\mathrm{Mc} / \mathrm{s}$ absorption nor strong $85.5-\mathrm{Mc} / \mathrm{s}$ discrete source. In the absence of absorption, the ratio of the two temperatures would be expected to be about 50 (taking $T \propto f^{-2.65}$ ). It is seen that for latitude +3 degrees the observed ratio is not much less than this, except near 360 degrees as pointed out above. We conclude that except in comparatively small areas absorption at this latitude must be small. There can be very little $\mathrm{H}$ II at 4 degrees from the galactic plane; if it is concentrated near the sun the EM cannot be more than about 160 .

Near the galactic plane (the -1-degree curve), the ratio is very much lower than 50 over a wide range of longitudes that corresponds to the "trough" on the records that was mentioned in the introduction. We shall now consider

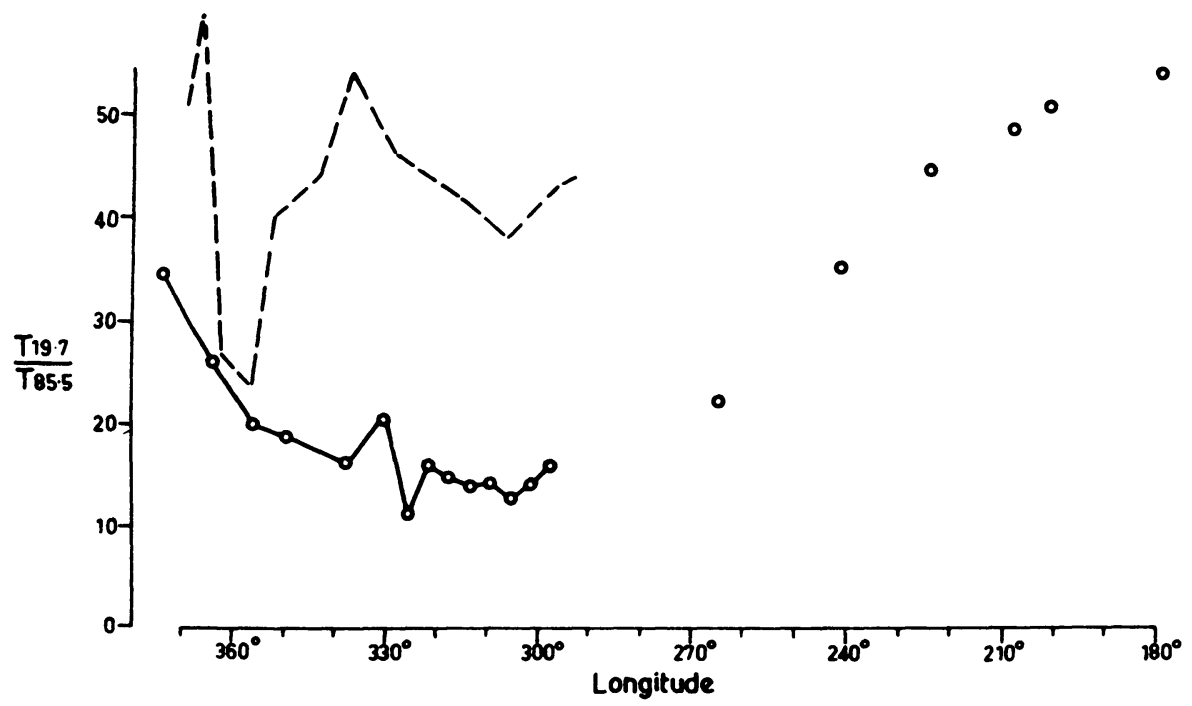

FIG. 4. The ratio of $19.7-$ and $85.5-\mathrm{Mc} / \mathrm{s}$ aerial temperatures as a function of longitude. Dashed line: latitude +3 degrees; circles : latitude -1 degree (avoiding strong sources and 19.7-Mc/s minima). 
whether it is possible to account for these low values by $\mathrm{H}$ II absorption alone, or whether it is necessary to invoke some change in the spectrum of emission near the galactic plane. For our model of the Galaxy we combine Mills's model of the nonthermal emission with a distribution of $\mathrm{H}$ il consistent with Westerhout's observations.

In the model we assume that the sources of nonthermal emission away from the central regions of the Galaxy are practically confined to Mills's three spiral arms, and that each arm contributes $70,000^{\circ} \mathrm{K}$ to the brightness temperature. The $\mathrm{H}$ II is also assumed to be confined to these spiral arms with the $\mathrm{EM}=800$ for lines of sight traversing one arm at distances less than 30 parsecs from the galactic plane, and $E M=200$ for lines of sight between 30 and 100 parsecs from the plane. The $\mathrm{H}$ II is also assumed to be distributed, on the average, along the center line of the arms, and the total amount of $\mathrm{H}$ II is made to fit Westerhout's data. (This distribution is rather different from that proposed by Westerhout, who derived a model showing a continuous distribution of $\mathrm{H}$ in between 3 and 10 kiloparsecs from the galactic center. However, Baade [12] has shown that in $\mathrm{M} 31$ the $\mathrm{H}$ II is closely confined to the outer spiral arms, and Westerhout's distribution is no doubt affected by the assumptions made in his analysis.) Because the angles they subtend at the sun are so small, the spiral arms on the far side of the galactic center produce only small attenuation.

Without going into the details of the computation, the result shows that for longitudes near to, but a few degrees away from, the galactic center, the $19.7-\mathrm{Mc} / \mathrm{s}$ temperature should be just over $200,000^{\circ} \mathrm{K}$. This agrees well with the value of about $250,000^{\circ} \mathrm{K}$ actually observed, and we conclude that absorption in $\mathrm{HII}$ is sufficient to explain the low values of $T_{19.7} / T_{85.5}$ near the galactic plane, without invoking changes of spectrum. Very near the center the problem is more complicated; it will be considered below.

Since the $\mathrm{H}$ II in the nearest spiral arm fills a greater fraction of the aerial beam than that in the more distant arms, it makes a greater contribution to the attenuation. As we move away from the longitude of the galactic center the distance to this arm increases steadily and the attenuation should decrease. Fig. 4 shows that this is so; the apparent attenuation decreases rapidly between 360 and 10 degrees where the line of sight is about tangential to the spiral arm. In fact, there are small, but probably significant, changes in the slope of the curve at about 300 and 355 degrees, which would correspond to the passage of the $\mathrm{HII}$ in the next spiral arm out of the aerial beam. (If, as supposed, the distribution of the sources of nonthermal emission are the same at $19.7 \mathrm{Mc} / \mathrm{s}$ and $85 \mathrm{Mc} / \mathrm{s}$, the ratio $T_{19.7} / T_{85.5}$ should be constant, in the absence of absorption, in spite of the sudden change in the amount of nonthermal emission as the line of sight becomes tangential to a spiral arm.) There are too few observations at present to know just what happens in the range 250 to 290 degrees, but it does seem that Fig. 4 fits in well with the hypothesis that the $\mathrm{H}$ II in the Galaxy is confined to the spiral arms.

Returning to the center of the Galaxy, it will be seen in Fig. 4 that the 
point for longitude 326 degrees, -1 degree is lower than all the others. This corresponds to one of the nonthermal peaks of $17 \mathrm{~S} 2 \mathrm{~A}$ at $85.5 \mathrm{Mc} / \mathrm{s}$, and the relative intensity at $19.7 \mathrm{Mc} / \mathrm{s}$ is reduced by the absorption in the thermal component of this source. On the other side of the source, the point at 330 degrees is actually higher than the neighboring points. At this longitude there would be little, if any, attenuation from the thermal component of $17 \mathrm{~S} 2 \mathrm{~A}$, but there will still be the foreground attenuation from the spiral arms. The high value of the ratio of temperatures therefore means that, at least in this direction, there is a change of spectrum (perhaps in the source) or else a "window" in one of the spiral arms. This situation makes difficult any attempt to use the $19.7-\mathrm{Mc} / \mathrm{s}$ absorption data to estimate the distance of the thermal component of 17S2A. Before any conclusions can be reached, a detailed analysis is required of all the 19.7-, 85.5-, and 1390-Mc/s data covering the region of the galactic center.

\section{H II ABSORPTION OF DISCRETE SOURCE RADIATION}

Since some of the $\mathrm{H}$ in regions are completely opaque to $19.7-\mathrm{Mc} / \mathrm{s}$ radiation it would be expected that at least some of the discrete sources observed at $85.5 \mathrm{Mc} / \mathrm{s}$ would not be observed at $19.7 \mathrm{Mc} / \mathrm{s}$ even if the flux density increases with wavelength. This is found to be the case, a striking example being the nonthermal source very close to $M 20$ (number 28 in Westerhout's list [3]). Assuming that the spectrum of the source is the same as that of the general galactic radiation, and making due allowance for the difference in aerial beamwidths, it would be expected that the excess brightness temperature due to a source of small angular size would be some 15 times greater at $19.7 \mathrm{Mc} / \mathrm{s}$ than at $85.5 \mathrm{Mc} / \mathrm{s}$. On this basis, the source near $\mathrm{M} 20$ should produce an increase in aerial temperature of over $250,000^{\circ} \mathrm{K}$ at 19.7 $\mathrm{Mc} / \mathrm{s}$. However, it will be remembered from the earlier sections of the paper that in this direction there is actually a deep minimum in the aerial temperature (see Fig. 1), and the temperature observed, $220,000^{\circ} \mathrm{K}$, must include at least a very large proportion of general radiation. We can therefore say that this source must lie behind $M 20$, at a distance greater than 1300 parsecs.

For a full study of the effect of $\mathrm{H}$ II absorption on the intensity of the nonthermal sources, the detailed brightness distribution around the galactic plane is required. It should be possible to put crude lower limits to the distances of a number of sources (for example, the number of spiral arms between a source and the sun), but more important would be to pick out the few sources that might appear in front of $\mathrm{H}$ II regions, 'thus having upper limits to the distances. This would give upper limits to the linear dimensions of the sources and to the total power actually radiated. It would also be helpful for optical investigations to know definitely whether a particular source was nearby or far away.

\section{REFERENCES}

[1] Shain, C. A. Aust. J. Phys. 10, 195, 1957.

[2] Gum, C. S. Mem. R.A.S. 67, 155, 1955. 
[ 3 ] Westerhout, G. B.A.N. 14, 215, 1958.

[4] Johnson, H. M. Ap. J. 121, 604, 1955.

[5] Sharpless, S., and Osterbrock, D. Ap. J. 115, 89, 1952.

[6] Blythe, J. H. M.N.R.A.S. 117, 652, 1957.

[ 7] Rishbeth, H. Aust. J. Phys. 11, 550, 1958.

[8] Morgan, W. W., Stromgren, B., and Johnson, H. M. Ap. J. 121, 611, 1955.

[9] Morgan, W. W., Whitford, A. E., and Code, A. D. Ap. J. 118, 318, 1953.

[10] Mills, B. Y. Paper 79.

[11] Mills, B. Y., Hill, E. R., and Slee, O. B. The Observatory 78, 116, 1958.

[12] Baade, W. A. Univ. Mich. Obs. Publ. 10, 7, 1951. 